\title{
FROM DESCRIPTIVISM TO CONSTRUCTIVISM - A CHALLENGE TO SYMBOLIC MODELING. II
}

\author{
A. KJELLMAN \\ Dept. of Regional Planning, Royal Institute of Technology, Stockholm \\ Dept. of Information Technology, Mid-Sweden University, Sundsvall
}

E-mail: Arne.Kjellman@ite.mh.se

Received October 30, 1998

\begin{abstract}
Classical modeling is not isomorphic, on the contrary the "objects of reality" or the like is the source of a homomorphic mapping performed to produce the model - just the way a piece of landscape is portrayed by its map with some bewildering details left out. We are thus taught that this process of modeling (or abstraction) is a plain mapping procedure we call this descriptivism or representationalism. The prevailing object-oriented modeling approach - or realistic approach - has some serious shortcomings due to the negligence of some aspects of the observer function, which for instance has resulted in a "world definition" made from "outside" the living consciousness (realism or materialism). By reversing this picture instead taking off from the impressions arisen within the subject's (the observer/knower's) conscious experience - the subject-oriented approach and ask how a living consciousness organizes itself to handle the task of living, we gain new insights in the process of conceptualization and learning. We learn that the dualistic world view is superfluous and should better be replaced by a neutral monistic approach, where the hypothetical existence of an independent outside reality (realism) can be substituted by the idea of a reality constructed from inside a living consciousness nothing else but a model whose main purpose is to guide human anticipation and facilitate communication. Taking that stances the main tasks of human consciousness just become modeling - creating the outside model reality and the inside domain of feelings. In such a framework also the classical truth - in the sense of a God-given modeling truth become meaningless - and must be substituted by the Pierce-ain pragmatical or consensual truth. In the subject-oriented approach states, properties etc. are not given any observer independent existence. On the contrary they emerge at the moment of their measurement as advocated by the Copenhagen interpretation. Bell's theorem also states: Given the quantum mechanics, either the idea of Einstein locality or the idea of an observer independent reality must be abandoned. The subject-oriented approach clearly abandons the idea of a pre-given observer independent reality - in favor of a cognitive agent created private reality, which then become the base for defining an "objective reality" in the form of a consensual scientific agreement.
\end{abstract}


From descriptivism to constructivism - a challenge to symbolic modeling 99

\section{WHAT IS REALITY?}

Bell's theorem shows that quantum physics [3] is incompatible with the proposition that measurements discover some unknown but preexisting reality. This idea is most clearly pronounced in the so-called "Copenhagen interpretation" [4] and in the writings of post-Wittgensteinian philosophers like R.Rorty [5]. J.A. Wheeler [6] also rises the question as to whether reality is a great machine or depends on "an idea so obvious that it is not obvious?"

The subject-oriented approach gives pre-eminence to the latter suggestion and we start out with the tentative answer that "reality" is given when we contemplate the subset of all sensations that we do not consider to be part of ideas, dreams, hallucinations etc. We say such sensations come from "reality" - and that the impressions formed by such sensations - percepts - are in a sense more "real" than our dreams and the like. The decisive quality we are talking about in that respect is permanence. Thus we assume todays Eiffel tower is also tomorrows Eiffel tower both in its appearance and meaning.

Conscious experience is then intellectually built up as an ordered set of impressions - percepts ("coming from reality") and non-percepts (like feelings, dreams, hallucinations etc.) A closer investigation reveals that the phenomenon we call "reality" is the subset of sensations and impressions qualified as "real" by scientific investigation. Physics is principally the judge of what is "real" [7] or not - but the elements of "reality" are nowadays not necessarily classified as material.

Let me call this "outside reality" the "out-there-ness" and make a clear distinction between this and another more vivid and close at hand reality the "inside reality" (I-reality). The latter is the colorful and vivid apparition (the process) emerging in our conscious experience when watching the "outthere-ness". The question is now, what is the relation between these two realities - and which one do we refer to when we use the single term reality? My claim is that the only phenomenon worthy of that denomination is the I-reality - and that this very personal and private model is the only sign we as living beings have of the "out-there-ness".

As already pointed out I-reality is not even a homomorphic mapping of something "out there". No - I shall even at this stage propose that this is a model is a construction built on the clues from outside 1 - the "out-there-ness" - and this model construction reveals more about the working of our brains than any "physical" feature of the "out-there-ness".

The prevailing object-oriented approach to science tacitly rests on a daring hypothesis [8] - the preexistence of a "reality" which has definite properties, whether or not it is observed by someone. To the existence can I agree - thus rejecting solipsism. This only amounts to a feasible definition of existence. But my claim is that this presumed "out-there-ness" couldn't rightfully be assigned states and properties that is observer independent; on the other hand, states and properties may be assigned to the I-reality. But if we wipe away

${ }^{1}$ The Peirce's indices -i.e., "physical" connected. 


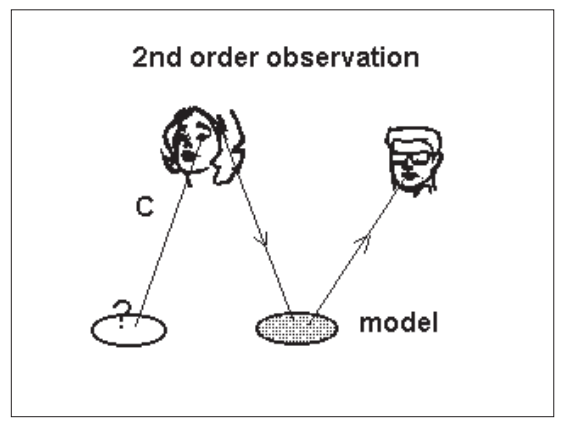

Figure 1.

all observers then there is no I-reality to which properties and states may be assigned. Thus the notion of I-reality make sense only in the presence of an observer just in the same way any dream is in need of a dreamer. How is it then possible to speak about the observer independent properties of reality?

To avoid the "trap of materialism" we simply avoid postulating any "outside" reality, for the simple reason that we have neither any proof of its existence nor the slightest idea of its essence. Therefore let us mark a phenomenon (object) in the "out-there-ness" by a question mark (?). The woman in 1 is an observer looking at the "out-there-ness" - and this is the crucial point. The objects of "out-there-ness" are unseen - all what is "received" by the observer are the clues from outside.

Let us say that she sees a tree. Where is the tree - in the "out-there-ness"? No, the tree (the object) emerges in her conscious experience and the only sign we have about this "existence" is her spoken words "I see a tree". When I look at the same question mark (?) I also see a tree. But how can I be sure that her percept is similar to mine? I simply cannot - and what is "similar" supposed to mean in such a context?

So the only sign I have on her conscious experience is her utterance "I see a tree" - the model - and this act of communication are due to the spoken language. To utter words (assemble) from a given language, or draw symbols (assemble) from a given graphical language or the like is an outward action [1] of an observer/actor and this is the only means to communicate different ideas or concepts to other consciousnesses. Such models are laid out in different modeling frameworks (different languages) and the end product - the model - is a re-presentation of the original presentation (percept) in the conscious experience of the observer/knower. Observation does not even have to involve any "out-there-ness", as for instance when an artist paints the experience of a dreadful dream.

In order to be useful the result of this outward action - the model - has to be "caught in the fly" or laid out (permanent-ed) on some medium of permanence thereby establishing a memory function - and suggesting that communication and memorization [9] is one and the same process. Even the 
"aether" can be regarded such a medium and the electromagnetic light wave then become the "printed matter" stored in such a medium. The medium in use serves as a base of data and the receiver of the message delivered by this medium can be another consciousness (interpersonal communication) or the self (autocommunication [10]). In this situation we must carefully take the permanence factor of the medium used in consideration.

Thus any model or part of a model (sign, symbol or representation) thereof must be considered an observers/actors action - but the reversed is clearly not applicable. The modeling path thus is very dependent on human constructive fantasy and has always been regarded as constructive, even in the classical sense of modeling. The spoken language and mathematics are the outstanding representatives indicating this state of affairs.

The need of activity from the receiving part is also obvious - firstly to observe the model (or eventually go and look for it) and secondly to interpret it. Behind this instantaneous act of interpretation is something considerably more comprehensive - how to learn the art of interpretation - which is so essential for both the physical and social survival of the individual.

\section{Observation and measurement:}

Vision is the far most important human observation capacity and is the perceptual capacity far most investigated [11] and best understood. We must also look at measurement from a modeling perspective. For an observer to measure some "property" it firstly has to be "separated" or "abstracted" from the phenomenon under consideration. Then the sensory impression has to be assigned a numerical value according to some scale and standard unit of measurement and presented outwards. An observer performs these two steps in parallel. This is a modeling task - not an easy one - but nevertheless a plain modeling effort. For instance the length of an object is measured by separating the spatial extension (foreground) in some direction from the rest of the percept (background) and then a length measure is assigned to it by the comparison with the standard meter. Such a "naked eye" measurement is highly "subjective" and uncertain and therefore the natural sciences depend on measuring devices and sticks.

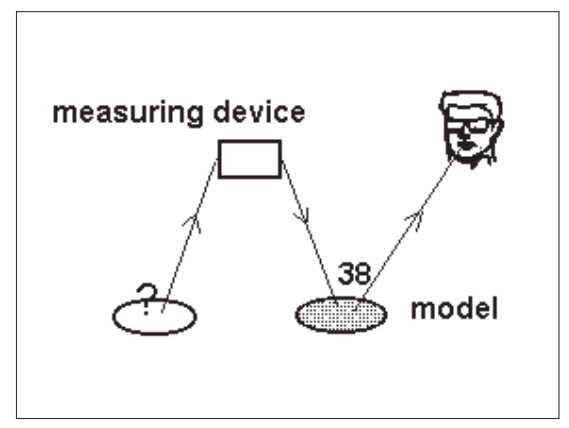

Figure 2. 
To construct a measuring device we have to find a "physical" phenomenon which separates the "property" (foreground) from the rest of the phenomenon (background) and then to measure ${ }^{2}$ the "intensity of the corresponding sense impression" by finding a measurable correlated "physical" phenomenon. Once such a device is invented and mechanized, the non-stable human judgment can be replaced by the output of a designated, warranted measuring device. Once, we as inventors, have found the proper "property abstraction" we can depend on the measuring device, which is a well-defined transducer. Thus making measurements turns out to be a true mapping procedure - the original signal $S$ is modeled by its output $f(S)$ in an act of transduction $S \rightarrow f(S)$ in the mathematical sense. This act of transduction is repeatable to a high degree - this is the crucial point - otherwise the device has not been duly warranted as a measuring device.

Unfortunately the use of measuring devices does not "objectivize" the measurement process. A measuring device can be used to extend the senses of man - to make him see "invisible" phenomena. This must not be confused with "objectivity". A measuring device is due to its mechanistic/electronic features more stable and repeatable but a human being. It is also more reliable but a human observer in another sense - since it is invented and tested by a certified engineer - we might call him an expert observer. In the same way we can recognize other expert observers - we title some of them experts or professors. But this state of affairs must not either be confused by "objectivity" in the sense of an observer independent reality.

When we compare 1 and 2 we realize that the observation of a measuring device is nothing more or less than a $2^{\text {nd }}$ order observation. The only difference - but a crucial one - is that the living observer (the constructor) has constructive power and the ability to self- adjust, abilities that are totally missing in the classical measuring device (the transducer).

\section{OBSERVATION IS DECISION}

In the light of what is said the materialistic conceptions of state, property and object stands out as ill defined - and this is one reason to instead make use of a subject-oriented approach. This latter suggests that objects have no properties except when measured and that "property" is a quantified human sensual impression (expressed in bits) outward projected onto the object measured. The essence of this "property" is nothing else but the biological reaction met with by the peripheral sensors - i.e. it carry no other "qualities" but an address to the peripheral sensor engaged and a measure in bits called the "experienced sensual impact" (quantity) - which is attributed to the sensory signal. From an intellectual point of view this essence is irreducibly self-referential, requiring information about the "address" of the peripheral

${ }^{2}$ Measurement procedures are in general more elaborate - but this principles apply. 
sensor involved and in consequence mainly a "property" of the observer. What is further behind this sensual impact (the ontological question) is out of the reach of human intellect.

Another reason to use the subject-oriented approach is to cut off the infinite regression of observers watching other observers; a fact most clearly pointed out by von Neumann [12]. A very reasonable choice is to put our trust in the autonomous unity of the human being and place the cut at the level of the subject.

In doing so I will claim the essence of observation is change [6] - a mental state transition; When your conscious experience undergoes a mental state transition then you observe something - and this state transition is in turn attributed (or projected outwards) - onto something "outside" your conscious experience [13]. Here is the crucial point of the subject-oriented approach - the outward projection - that closes the circular loop of causes. This process goes on unconsciously - the processes occurring in human brain appears to be located somewhere else (projection) - and outside consciousness, thus reminding us about the words of Wheeler "an idea so obvious that it is not obvious?"

Here is not the place to explicate on mental state transitions, and to explain just let us agree on that a "car" is nothing else but a set of coherent mental state transitions. Schematically the process of observation/projection then can be explicated:

a) conscious experience sees a car

b) the I-reality is outward projected

c) the I-reality "disappears" - was actually never recognized as a local phenomenon

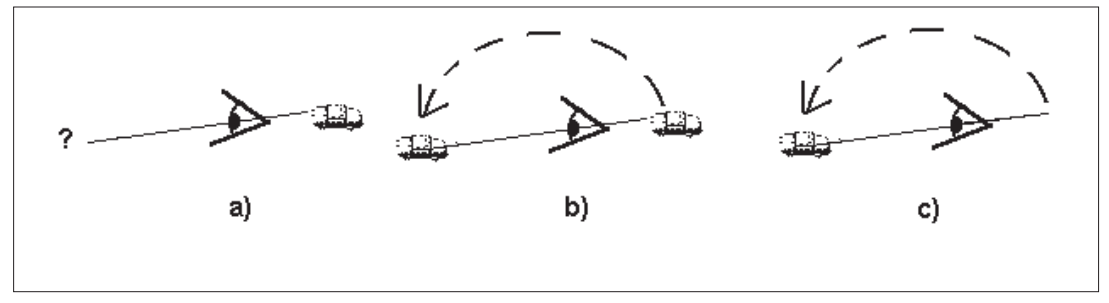

Figure 3.

When there are no mental state transitions - there is no observation regardless of what has "actually happened outside". Observation is nothing else but an act of decision - the decision that something has changed within your conscious experience - and decisions are made by knowers. When it comes to observation the observing subject is the decider/knower. As living beings we have to learn to separate prospective harmful state transitions from the harmless ones. Once we have learned our lessons these acts of decisions 
that are quite straightforward, but the amount of learning to reach that state of knowledge will have been comprehensive. From that point of view there is certainly no need for any colorful "out-there-ness" - the I-reality is all that we need.

Since we do not believe in solipsism (bare mind-spinning) the observed state transition is hypothetically attributed to something coming from the "outthere-ness" or from the bodily "in-there- ness" (feeling and imaginations) but from "outside" conscious experience anyhow. Thus the momentarily classification of a percept is a very private process - and the "objective" features of this process is due to nothing else but the individual skill, which are the result of upbringing, education and earlier experience.

Perception seemingly consists in events when the so-called "physical world" undresses (reveals itself) and shows up in bits of information [14] to the individual conscious experience. Raw data (nerve impulses) become information inside the interpreters consciousness - after the act of interpretation. Consequently bits of information or messages are "stuff" that are born inside a living consciousness - and dies outside it. No meaning (or substance) can be attributed to such raw data outside a knowing consciousness. In this way the need for the classical real/abstract dualism vanishes - to be replaced by a monistic view - where the inputs of consciousness are bare data (impulses). This is to say that data without associated interpretation procedures (contained in an interpreting brain) are just useless data - or garbage.

Raw data emitted by "external objects" then become information inside the interpreters consciousness this is the picture advocated by the object-oriented view. But the idea that the brain adds raw data along the perceptual path on a private basis is merely a twist to try to rescue objectivism. How do we tackle the problem with hallucinations showing green Martians coming through the wall, when there is no way for a conscious being to objectively separate "perceptual" data from "non-perceptual"? In this task he is certainly directed to clever guesses and useful guesses are clever guesses - this is the core idea of Peirce's pragmatism.

\section{CONSTRUCTIVE ELEMENTS}

The cognitive sciences has clearly indicated that the brains function is constructive i.e., there is information in conscious experience that have no correspondence in the "out-there-ness" - according to the collective convention used.

This is the first challenge symbolic modeling. A 3D impression in the figure outwardly projected onto a plain sheet of paper reveals that data are "constructively added" between the eye and consciousness. Such a constructive functionality cannot be handled by a plain mapping. The blind spot of the eye is also unseen - which also confirms this "filling-in activity" of the hu-

man brain. We are also familiar with the optical illusions produced by the Nectar cube, where we are able to choose an interpretation at will. In the 


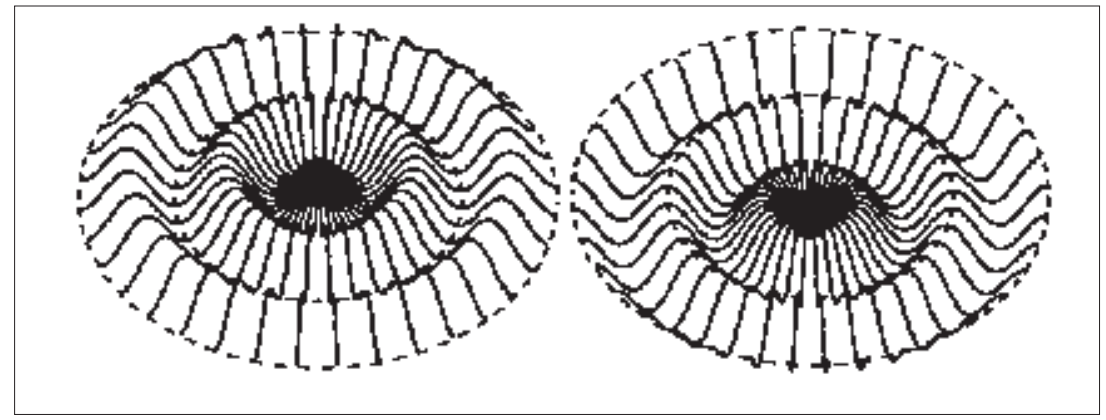

Figure 4.

literature on psychology and cognitive science there are numerous examples on such phenomena. Such "corrections" are true constructive acts performed by a highly adaptable brain. Here the affirmative differences between descriptivism (transduction) and constructivism (construction) clearly stands out. In both cases raw data are removed (to avoid processor overload) - but in the latter case the brain adds information which seemingly has no sensory counterpart. By this manner of speaking, we are unfortunately still stuck in the object-oriented view, since the "corrections" performed are just corrections in the object-oriented sense.

This inevitable means that we run the risk that every observation contain subjective elements - i.e., that the constructive brain has inserted raw data to complete the information missing according to its own private opinion on the path from the senses to conscious experience. There is certainly no way to eliminate such raw data insertion on the perceptual path until we can observe "directly" by conscious experience.Admitting this state of affairs makes any observation private and the picture of an objective science based on objective observation fades.

\section{THE SUBJECT-ORIENTED APPROACH}

According to the subject-oriented view - observation actually works the other way around. Outgoing from acknowledged state transitions in conscious experience our intellect constructs an outward path to a reason in the "out-thereness". This view reveals that the "outside world" is epistemologically created from the "inside". This act is not re-constructional. This is a process of construction - since epistemologically there is nothing to re-construct. When a mental state transition has proven useful to us we have learn to project it outwards onto the "out-there-ness" (a path originating in the mental state transition and ending up somewhere in the "out-there-ness") - this is the essence of observation in the subject-oriented view. We see by consciousness and not by the eye - our consciousness paints a useful "out-there-ness" by pro- 
jecting the privately experienced internal mental state transitions outwards.

On the other hand, when a mental state transition proves to be useless, we "forget about it" and adaptively cease to observe it in future. That is to say, mental state transitions that are of no practical use to a cognitive agent are ignored until the point of disappearance - a process of submergence.

Such an I-reality should not be called a re-presentation, precisely for the reason it has not even been presented to anyone before. The correct denomination is a presentation constructed on the basis of clues from outside - and in that respect "mental" models (endo-models) differ from "physical" models (exo-models). "Physical" models of the classical reality are re-presentations of the content of conscious experience - which itself is a percept - i.e., the first and original presentation of the presumed "out-there-ness".

The next step is to realize that the outward projected model - the I-reality does not even portray the "out-there-ness" - it is mostly a perceptual construct (the I-reality). The only demand we have on this perceptual construction is that it should allow us to effectively make environmental predictions.

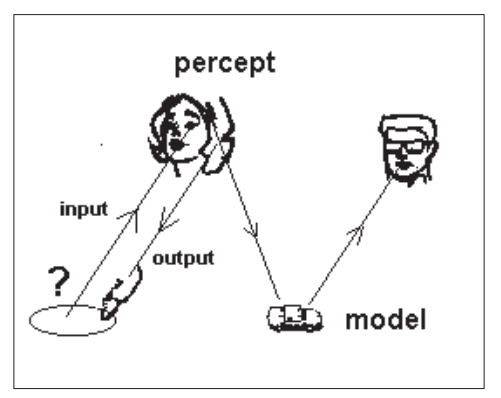

Figure 5.

As seen from the 2nd order observers perspective (the scientist's) the "outthere-ness" is virtually a "black-box" - the output of which is the subject's percept and the input is the subject's action. The I-reality (the image) is the subject's tool to control its outward actions, but the scientist does not see this "image". All the scientist sees is his own I-reality - which includes the subject - but certainly not the subjects I-reality (image).

Let us ask what happens if the subject looks at a phenomenon which is "invisible" - an atom for instance. Since the brain does not produce any perceptual construct (I-reality) in this case, the subject is free to invent an arbitrary model - within the constraints that it should be the good base of predictions. In that respect anything goes [15] - but needless to say some models work better than others do. Since we use this model when communicating our experience to other beings a model featuring some everyday phenomenon will be appropriate, since most human beings have gained an immense experience from the happenings of everyday phenomena. In short we make use of analogical thinking and modeling [16]. I will claim this is why 
the atom is seen as a miniature of the solar system, the particle a grain of sand, the electromagnetic wave the billow surface of the ocean etc.

\section{THE HOLISTIC VIEW}

The I-reality is projected outwards onto the estimated "location" and we as human beings have not the slightest feeling or internal indication of this process of outward projection going on in our own conscious experience. I believe that this is the reason why the intellectual [17] understanding has taken a long time. In this view there is certainly no images in the mind of the observer - just a process going on in its brain but the "feeling" of this process is subjectively located "outside" the brain to find the anchorage (the clues from outside) - this is the projection. And this is also the proof of the "out-thereness" - i.e., the usefulness of the outside clues. To this conclusion we have come without any reality postulation in the materialistic sense of the word.

In this view visual impressions are a just bare feelings to be treated at the same level as other feelings. We know that this outward projection mechanism has been recognized on the non- perceptual level for many a years, for instance in the psychoanalysis - in this case a living being projects its own fears and feelings onto the "object" of observation.

Let us explicate on the constructive build-up: The colorful images (I-reality) emerging in a newborn child's mind is as first highly confusing. By the movement of its hand at the same moment the child almost accidentally happens to produce a tactile input. This tactile input becomes the "clue from outside" which is experimentally correlated to the mind image. As time goes by, the perceptual impressions that can be successfully (criterion: usefulness) correlated to some tactile impression are established (permanent-ed) in the form of recognizable images (the objects of I-reality). To be of use this "image" must be outward projected upon the "clues from outside", thereby defining "something" "outside" consciousness and at the same time defining spatial distance as the measure of the arm's motor movement. The impressions that cannot be brain-computationally be correlated to any permanent phenomenon are useless and are in consequence suppressed by brain in order to avoid cognitive confusion. In this view the colorful images of I-reality are thus used to dress the "invisible clues from outside" producing a vivid and colorful reality (by means of outward projection).

In consequence the I-reality (image) is the cause of the search for a "clue from outside" and such an outside clue (when found) is the cause of the cognitive establishment of the object. Any object definition is then due to a circular process of mutual casual reinforcement. In such a process we can manage without neither the images of I-reality nor the clues from outside and this necessitates a holistic approach.

We can say that the outward projection mechanism explains why the braininternal processes (the I-reality) appears to reside "outside" consciousness and there become the colorful REALITY - which is superimposed upon the clues 
from outside (the "out-there-ness"). In that respect reality with its properties is the model of the "out-there-ness" - the clues from outside, which cannot be attributed states or properties or define objects or systems. The colorful REALITY is the result of our nervous tissue reaction on reflected light - of course the bat's REALITY will be "different" because of the difference in its brain structure and of the properties of the reflected ultra-sound. The properties of REALITY cannot possibly be independent on any observer in such a setting. In consequence not even the geometrical properties of I-reality are unaffected by the observer - that is easily realized when we assume an observer having eyes sensitive to $X$-rays exclusively.

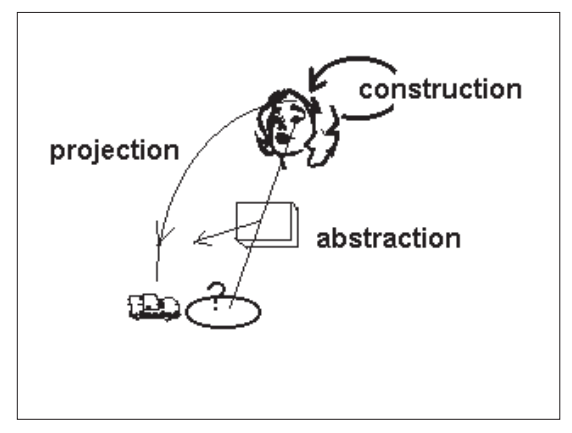

Figure 6.

In conclusion not only the modeling path - is constructive, but also the perceptual path. The modern findings of cognitive science have clearly paved the way for such a view. Using the object- oriented approach data are constructively added between the eye and consciousness or according to the subjectoriented approach we find some data in conscious experience that have no "outside explanation" according to the objective consensual agreement in use. In both views this is the result of the doings of a constructive brain - and the prevailing opinion of the mapping procedure performed by the human perceptual system is now in decline. This constructive process along the perceptual path, which cannot be captured by the traditional mapping procedure, is a true challenge to symbolic modeling in all its forms.

Descriptivism turns into constructivism in a shift of paradigm in a true Kuhn [18]-ian sense. The purpose of science is not any longer to portray some "out-there-ness". The purpose of science now become to explain how we from inside learn to cope with a changing environment in spite of its anonymity and how we construct and make use of the models that make us (and our brains) so extremely successful in ours acts of prediction, control and communication. 
From descriptivism to constructivism - a challenge to symbolic modeling 109

\section{WHAT HAPPENS TO THE TRUTH AND THE OBJECTIVE REALITY?}

The ontological question remains and we must ask what has actually "happened outside". Of course there is nobody to tell us any truth about that and this state if affairs already Kant [19] pointed out. The truth is hidden to any living observer - so unless there is a God to tell us the truth the classical interpretation of truth vanishes in this modeling context. There is not anything to compare the I-reality to, since the structure of the "out-there-ness" cannot justify any such comparison ${ }^{3}$. When a perceptual impression $S$ occurs in conscious experience and is outwards projected onto some constructed "outside-the-body reality" or some other "outside-consciousness" phenomenon then the "truth" of this state transition is wrapped in obscurity - and is actually beyond the capacity of human knowledge. All the observer can do is to try to verify $S$ by experimentally provoking similar transitions but this is a quite tedious task.

The observer can also check for the occurrence of other mental state transitions that are intellectually correlated to $S$ as a mean of verification (i.e., use other senses). The more evidence of a stable, repeatable and multi-faceted phenomenon behind $S$ - the more "real" $S$ is considered. The idea of an ontological truth vanishes in this setting - mental state transitions have counterparts in the "out-there-ness" only according to some probability - the more probable the state transition is the more "real" it is considered - but that is all. There is no way the observer - or any observer - can assure himself that "he is $100 \%$ right". He must believe in its own interpretations skills - or ask for some outside assistance to verify the probability of the $S$-occurrence.

Since the ontological truth has disappeared there is only way to define an objective reality. The concept of objective reality stands for a "reality" that can be use as a base in intersubjective discussions - i.e., a consensual basis. Since the ontological truth has vanished such a state of consensus could not be attained by reference to some "common outside reality". Instead we must rely on an inter-subjective agreement on what is to be regarded as objective knowledge - as touched upon in sec. 2 (I). This results in that the concept "objective reality" become a set of scientific models - which is "unseen" by any single observer and does not refer to any visual impression. This is then a consensual structured world-view - a single paradigm adhered to by a scientific community in the Kuhn-ian sense, which also lays out the rules of the activities of the normal science. In the subject-oriented approach the only criterion we put on this paradigmatic set of models and the activities of science is that they are useful. Scientific progress is then the procedure of replacing "old" models by fitter ones - but such an endeavor is of course heavily based on the goals of mankind. So the prevailing classical truth-criterion referring to some absolute truth (or observer-independent reality) must be substituted

\footnotetext{
${ }^{3}$ If a comparison was possible - who should perform this comparison when there is just the subject that "sees" the I-reality?
} 
by the Peirce's pragmatic and consensual truth criterion.

\section{OBSERVER CHAINS AND THE ULTIMATE CHALLENGE}

There is another challenge waiting for symbolic modeling and even for mankind's view on scientific practice. In order to explain the process of perception the constructivistic ideas mentioned in this paper must clearly be incorporated in the framework of cognitive sciences. In this context the scientific observer must intellectually construct a chain of causes from inside and out thereby identifying a chain of observers - or a series of observing agents ??.

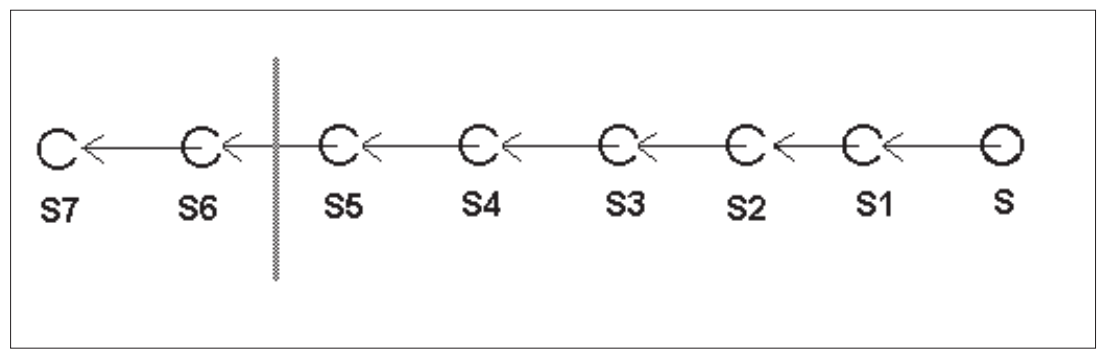

Figure 7 .

Taking off from an experienced state transition $S$ in conscious experience the human intellect must invent a casual chain of sub-observers [2] outgoing from this initial transition $S$ to establish an anchorage in the "out-there-ness". Something like - conscious experience decides a state transition $S$, which is attributed (connected) to a brain structure transition $S 1 . S 1$ in turn is the result of a state transition in the optic nerve $S 2 . S 2$ is the optic nerve transition connected to a retina transition $S 3 . S 3$ is the retina transition connected to a rod transition $S 4 . S 4$ the rod transition connected to chromophore transition $S 5$. $S 5$ the chromophore transition connected to a photon impact $S 6$. $S 6$ the photon impact is the result of an inter-atomic transition $S 7 . S 7$ is the result ... and so on - in another endless von Neumann "causal chain" - a similar observer hierarchy earlier pointed out. Now this hierarchy is expanded in the downward direction into a series of sub-observers - and every sub-observer is responsible for its part of the raw data processing. The physically boundary of the human body occurs at the level $S 5-S 6$. Parenthetically this point of view is easily expanded into the ideas of neural networks.

Cognitive scientist physicists claim that this observational path must have a bottom [20], [21] in such a chain - but from a subject-oriented point of view there is no need for such a bottom nor can it be defined. From an ontological point of view such a search is clearly also in vein - in spite of today's keen search for an elementary particle. From a modeling point of view (epistemological) we must pursue such a search until the level that we have developed a workable and communicable model that 
are firmly anchored in the "permanence of the out-there-ness" but there is no need to go any further.

For the sake of prediction we can theoretically also even eliminate or re-route all the immediate steps between conscious experience $S$ and the inter-atomic transition leap $S 7$ and still have a workable causal explanation left - a workable model of the presumed "out-there-ness" in its pragmatic sense. It is clear the path from $S$ to $S 7$ is a construction outgoing from the transition $S$, but nevertheless we might think that every step in this chain can be scientifically studied in such way that we can reconstruct what state transitions are "added or removed" in the object-oriented sense. This is not so - because the pathway from $S 5$ to $S$ also concerns the constructive parts of the pathway of the observer/experimenter himself. This pathway makes all his observations constructive and the pathway $\mathrm{S} 5$ to $\mathrm{S}$ can never be removed by any observer in any process of observation whatsoever. The only way to change or effect this pathway is by means of habituation, training and education. So in this setting the deductive power of man's observation is lost forever. Well, it was never there - and we must rely on induction - since induction takes in consideration the constructive interference undertaken by our brains. This is not only a challenge to just symbolic modeling - this is a challenge to all science.

\section{CONCLUSIONS:}

The subject-oriented approach has been suggested before but the time was not mature for its acceptance. The quantum theory has proven very successful but cannot coexist with the idea of a preexisting reality in its classical meaning. This is one reason to let go the idea of a preexisting "classical reality" and accept the suggestion that REALITY emerges in conscious experience. Then the pieces start to fall on their places - and we can still believe in a single common preexisting "out- there-ness" - but still mostly unknown.

The idea of a preexisting "out-there-ness" differs from the idea of a preexisting "classical reality" in one respect - the proposed "out-there-ness" has neither states nor properties. It will "exist" just because we say so - which then become the very definition of existence. The literally unseen "out-thereness" becomes a singleton - the anchorage of the clues from outside - and the emblem of permanence. This is the idea we can carry in our minds - but REALITY is the model the clues from outside.

Bell's theorem states that the idea of a preexisting "classical reality" cannot exist alongside the principle of local causes (Einstein locality) and the quantum theory. Since we have seen further arguments for that in this paper, let us abandon they idea of a classical reality and redefine the definitions of existence, state and property. Bell's theorem is of course valid for the proposed "outthere-ness", but I claim this pertaining just when we try to ascribe states and properties to the "out- there-ness". States and properties can be assigned to the useful and therefore pragmatically true inner REALITY only-here is the 
misconception.

These ideas support the Copenhagen interpretation that we cannot speak about a state and property until the observation (measurement) in question is actually performed - i.e., states and properties emerge in the inner REALITY only as the result of observations that is undertaken in order to visualize the "out-there-ness" on a personal level.

Contrariwise we should regard the "out-there-ness" as being constantly in flux and it can be assigned neither states nor properties - since these are bare observer constructions. Just models can be assigned such features - and REALITY is such a private model.

The need for any REALITY continuum also vanishes in this setting. The measurement process unavoidably takes time - thereby stating that any observation is by necessity discrete. So the observable REALITY is by necessity discrete [22]. The idea of a continuum is no law of physics, it is a mere principle stating that the classical reality was divisible to any fortuitous degree. Quantum physics has proven the limitation of this idea. This now applies to the "out-there-ness" - in virtue of an agreement - the quantum theory. On the other hand, now the imagined REALITY is divisible down to any fortuitous degree - it is just a matter of fantasy and usefulness.

This paper claims that REALITY is a model, but the miracle is not that the idea of a classical reality is gone. The miracle is rather how we in spite of that manage to construct individual realities that are so fit for control and communication and how we manage to adapt/adjust our brains for this important task. Education and up bringing is a mean marketing is another. The idea of an inside constructed REALITY is to me a very promising hypothesis - adding another dimension of responsibility thus: When we construct the world we live in by our own power - we are also fully responsibility for this construction and its appearance. This insight makes the prevailing ideas of the powers of market stand out in a peculiar light.

A materialistic, idealistic or psychedelic world - a cruel or a good world - the choice is mine, yours - and ours.

\section{REFERENCES}

[1] A. Rosenbluth and M. Wiener. The Role of Models in Science. Philosophy of Science, XII (4), Okt. 1945.

[2] B. Zeigler. Multifaceted Modelling and Discrete Event Simulation. Academic Press, 1984.

[3] P. Asher. Quantum Theory: Concepts and Methods. Kluwer Academic Publishers, Dordrecht, 1993.

[4] N. Bohr. Atomic Physics and Human Knowledge. Wiley, New York, 1958.

[5] R. Rorty. Philosophy and the Mirror of Nature. Princeton University Press, 1980.

[6] J.A. Wheeler. World as self-synthesized by quantum networking. IBM J.Res.Develop., 32 (1), 1988. 
From descriptivism to constructivism - a challenge to symbolic modeling 113

[7] D.J. Bilodeau. Physics, Machines and the Hard Problem. J. of Cons. Studies, 3 (5/6), 1996.

[8] J.F. Clauser and A. Shimany. Bell's theorem: experimental test and implications. Rep. Prog. Physics, 41, 1883, 1978.

[9] E. Fredkin. Digital Mechanics, North-Holland. Physica D , (45), 254 - 270, 1990.

[10] J.M. Lotman. K probleme tipologii kul'tury. Informations sur les sciences sociales, VI, 1967.(French transl.)

[11] B.M. Bennett et al. Observers Mechanics - A Formal Theory of Perception. Academic Press Inc., San Diego, 1989.

[12] J. Neumann. Matematische Grundlagen der Quantenmechanik. Springer, Berlin, 1932.

[13] M. Velmans. Consciousness, brain and the physical world. Philosophical Psychology, (3), 1990, $77-99$.

[14] J.A. Wheeler. Information Physics, Quantum. The Search for Links in Complexity. Entropy and the Physics of Information, SFI Studies in the Sciences of Complexity, VIII, 1990.

[15] P.K. Feyerabend. Against method: Outline of an Anarchistic Theory of Knowledge. Left Books, London, 1975.

[16] J. Holland et.al. Induction - Processes of Learning, and Discovery. The MIT Press, Cambridge, Massachusetts, 1986.

[17] von Glasersfeld. The Reluctance to Change a Way of Thinking. The Irish Journal of Psychology, 9 (1), 1988, $83-90$.

[18] T. Kuhn. The Structure of Scientific Revolutions. University of Chicago Press, 1962.

[19] I. Kant. Kritik der Reinen Vernuft. F. Meiner, Hamburg, 1781.

[20] J.J. Gibson. The senses considered as a perceptual systems. Houghton Mifflin, Boston, 1966.

[21] J. Fodor and Z. Pylyshyn. How direct is visual perception?: Some reflections on Gibson,s 'Ecological Approach'. Cognition, 9, 1981, 139 - 196.

[22] C. Fields. Consequences of nonclassical measurement for the algorithmic description of continuous dynamic systems. J. Expt. Theor. Artif. Intel., (1), 1989, $171-178$.

\section{NUO APRAŠYMO LINK KONSTRUKTYVIZMO - IŠŠŪKIS SIMBOLINIAM MODELIAVIMUI. II}

\section{A. KJELLMAN}

Antrojoje straipsnio dalyje tęsiamas ciklas darbų apie simbolinio modeliavimo taikymo ribas pažinimo procese. İvertinamas šiuolaikinių metodikų ribotumas, kai tenka spręsti kai kuriuos praktinius uždavinius. Analizuojamos objektinio modeliavimo taikymo ribos. Remdamasis įvairiais šiuolaikinès kvantinès fizikos reiškiniais, autorius vysto naują pažinimo modeliavimo metodiką. 